\title{
Surfaces and Shadows: Digital Shadow Puppetry and Augmented Silhouette Performance
}

\author{
Ian Grant \\ University of West London \\ Ealing School of Art, Design and Media \\ University of West London, Ealing, London W5 5RF \\ United Kingdom \\ ian.grant@uwl.ac.uk
}

\begin{abstract}
"Surfaces and Shadows" explores the interface between traditional shadow puppetry and tactile and embodied computer technologies. The work is illustrated with historical, theoretical enquiry, select case studies and documentation of original digital performance tools. The paper offers an extended definition of the digital puppet, discussing control interfaces, immaterially and the performance presence of the digital performing object. The paper presents a short exegesis of a prototype iPad application called the 'ShadowEngine' that makes multi-touchable digital shadow theatre possible using real-time physics based animation. I indicate design level insights and present potential approaches to making performance animation tools using a variety of touch and computer vision techniques. The author's ongoing DPhil in Critical Media Practice at the University of Sussex relates the media archaeologies of 2D and 3D puppetry, and in the present paper, augmented silhouettes, to landmark real-time computer animation work including compositions by media artists Myron Krueger, Golan Levin, Philip Worthington, Miwa Matreyek, Luís Leite, the author and others like Joon Moon and Design I/O. The paper will discuss related themes, including: the Cinematics and Kinesthetics of Digital Shadow Play Space; Mimetic Illusionism; 2D Puppet Forms in 3D Digital Space; Performer-to-character Mapping: 2D and 3D (dis)connections; and digitising material culture: kinetic play with heritage objects;
\end{abstract}

\section{INTRODUCTION}

To begin, there has been a ground swell of recent interest in and trending around digital puppetry. Part inspired by the readiness of the hackers and makers to construct demos that explore the ever increasing number of novel human interface devices and the ubiquity of creative coding tools that enable 'play' with them. The present rush of activity is also due, I would like to think, in part to the rich imaginarium of design, performance and cultural meaning that surrounds the 'puppet', in all it's manifestations.

As the 'puppet' is a hybrid and plural formation, the present paper selects and looks at shadow theatre and its importance in new media forms.

We have the Microsoft Kinect that has popularised whole-body interaction and computer vision techniques, walking in the ground laid by Myron Krueger in the late 1960s. The iPad, iOS and various Android devices have brought touch and tactile interaction to mass awareness, developing widespread explorations of the so-called 'natural user interface' (NUI), where we touch, grasp, move and shake our way to interacting with tangible and virtual objects. Gaining traction in developer communities, the Leap Motion: a gestural, finger and hand detection device, where each digit becomes a point of input, the orientation of the palm, the trajectory and pace of movement read in mid-air, in front of our screens. The potential attraction to the digital animator seeking embodied interactions with their creations is emergent. The attraction to the puppeteer is, I hope, very clear.

Such devices offer an opportunity for a radical remapping of the (whole) body or parts of the body as 'input'.

What is curious about them all is: each device is not interested in the surface detail of the body, but it's shadow, it's silhouette, it's capacitive aura. Each works by sensing and sampling a simplified image of the world (but like the ambiguity of shadows, a representation rich with expressive power). It is through this technological coincidence: that so much computer vision and sensor based work needs to transform the image of the world into silhouettes, that I am drawn to digital shadowography.

This paper aims to contextualise a current range of activities, all loosely interactive experiments, some consciously performance, others with potential as 
art or performance, forming a set of views about the emerging techne of embodied, tactile and teleremote digital shadow puppetry.

I hope to, tangentially, raise broader cultural and political issues raised by some technoappropriations of the visual form of lived traditional cultures. As we will see in some of the material, for example, in computer simulations of Turkish, Chinese, Indonesian or Greek traditional shadow theatres, digital remediation of past practices happen for a variety of reasons. The traditional practices are - all at once - bound to a sense of tradition and, at the same time, 'reinvented' and transform our memory (or amnesia) of historical media through a reintegration of form, image and apparatus, with the new.

\begin{abstract}
"... The notion of resurrecting dead media could prove farcical, futile, or more hopefully, deeply fertile. A broad accounting of the evolution of the apparatus, of the media image, of the history of the media effect, of excavating the embedded intellectual history, and so on, is surely the precursor of what will be an invaluable reconfiguration of a history largely focused on the device and its illusory images. Similarly, the rediscovery of uncommon or singular apparatus, novel and fantastic as they might be, is neither decisive nor fully adequate to formulate an inclusive approach that distinguishes it from connoisseurship, or worse, antiquarianism. Merely reconstituting or retrofitting "old" media into "new" contexts could, in this sense, only emerge as techno-retro-kitsch." (Druckrey 2008,
\end{abstract} ix)

As a loose creative method, media (an)archeology appeals to me. It aims, to paraphrase Druckrey, not to be reductive or dogmatic but to evolve histories within a larger scheme of reintegration of 'technologies, apparatuses, effects, images, [and] iconographies' (Druckrey 2008, ix).

I defend my work (often simulative, sometimes 'decontextualising' of historical puppetry forms) with full knowledge of the necessity for a 'critical technical practice', wishing to articulate the connections with living, dead and imaginary media of the past and enjoying the liberating sense of technological creative play and making.

\section{PUPPETRY CONTEXTS}

Puppetry and its rival digital puppetry are composite, hybrid performance practices. Though specific cultural traditions are central to understanding puppetry, its iconography and techne, each period adapts, transforms and reinvents traditions, for a variety of purposes: including (1) heritage preservation and the continuity of tradition and (2) experimentation and the pursuit of new forms.

"Contemporary puppet theatre is a rich and differentiated totality, taking in cultural elements of different provenance and from different epochs. (Jurkowski 1988, p.62)

The scale of puppetry in all its variety is beyond the scope of this paper: herein we focus on the digital puppet and the rich context of shadow. We should remember 'puppetry' is at once (1) the totality of performance traditions where objects are animated, and also (2) the puppet is a philosophical concept reaching beyond performance practice.

"Digital puppetry, the virtual counterpart of traditional puppetry, deals with manipulation and performance of animated figures and characters. Typically, performance and rendering occurs in real-time, which makes digital puppetry distinct from conventional character animation. Also, digital puppetry often includes novel interfaces for expressive control." (Digital Arts Week [DAW] 2007)

In some respects conventional character animation involves 'digital puppetry', when an animator moves a rigged character, that manipulation, in that moment, is an act of puppetry. So the DAW definition is a little limiting. Digital puppetry as I view it is a strange hybrid art form that presently includes a broad range of work and creative practices, for example: live real-time animation and performance capture processes; animation rigging, referring to animation performance systems like Pixar Studio's 'Marionette' software or the use of the entertainingly named 'dinosaur input devices'; kinetic art practice, involving servo/microprocessor controlled automata, robotic and animatronics; live multimedia performance; user-controlled characters and avatars; or, finally, tele-remote controlled lumia displays. Digital puppeteering may be a local affair or telematically distributed.

\section{CONTEXTS OF SHADOW PLAY}

Doing a service to the recent history and contemporary shadow theatre is beyond the scope of the present paper, but I wish to: provide aesthetically informed definitions of the cultural philosophical significance of shadows from Gross (2012):

\footnotetext{
"The thinnest of puppets, the poorest, the least substantial, is the puppet of shadow theatre -the true puppet here being the moving shadow itself, a thing bound to the screen, whose life is independent of the opaque or translucent silhouette of paper, leather or plastic that casts it. This is puppet all of surface, with no back to it, no depth, or only such hints of depth as are caused by the silhouette's being held closer or farther away from the screen, or with one shadow overlaying or passing through another. It is a shape which, when it fades away, recedes not so much into darkness as into light, always part of something larger than itself, something that shares the nature of what is seen and what is unseen." (Gross 2012, p125)
}

The early twentieth century saw a major challenge to shadow traditions in the form of cinema.

A multi-fold transformation occurred in shadow theatre in the modern period: the halogen lamp meant now the surface plane of the puppet performance was moveable in the z-axis and focus, literally, changed, the quality of shadow as volume, 3D forms and new materials introduce dynamism, colour and a new liveliness. Also, the light source could be mobile.

Through practical examples of digital shadow theatre, we see reorientations and transformations 
of screen space from tabletops, moveable projectors to mobile devices.

There is scope for a fuller contextual and technical description of the range of shadow traditions: each context - traditional, experimental, contemporary, pull on and transform the knowledges established through traditions of practice. In the digital space we at once see the reinvention and the establishment of new, innovative techniques.

\subsection{Characters, the Mimetic and a Liberation from Illusionism}

Philosophers of puppetry, of which Roman Paska is a luminary, raise questions that also resonate through the digital domain. Paska (2012) accounts the ever-increasing descent towards illusionism in digital animation, puppetry and cinema. He offers thoughts about character, mechanism and magic that outline how any object can 'become puppet' through performance, including, I add, digital representations.

Paska marks a conceptual distinction between illusionism and primitivism (in an aesthetic sense): illusionism attempts to be seamless. Illusionists seek unbroken moments. Those exploring primitivism:

\begin{abstract}
"... aim always for a series in which the illusion of being is consciously fragmented by the intrusion of awareness into the structural mechanics of animation, the real nature of the objects employed and the real time of theatrical activity. In the 'pure' puppet performance, focus oscillates between the real time and nature of the puppet object, and the illusory time and nature of the puppet as character sign. The puppet seems to 'come' alive without pretending to 'be' alive, with an effect closer to magic than technology." (Paska 2012, p.140)
\end{abstract}

In a way, through the technological simulation of ancient shadow forms we have a blend, a curious hybrid of illusionism and primitivism (in Paska's sense). He sees a similar hybrid future for puppeteers, in flux between contrary aesthetic forces: Enigmatically he states:
"Two futures or one: primitive illusionaries singing the body electric; illusionary primitives speaking in tongues?" (Paska 2012, p.140)

\section{CONTEXTS FOR DIGITAL PERFORMANCE}

The poetic and aesthetic considerations above raise numerous questions. In digital shadow performance are we just speaking of the simulation of existing forms? How are such concerns explored through a range of experiments within digital shadow puppetry? In the range of work surveyed simulative acts feature. How should they be studied? I follow Cubitt (1998):

'Every visualisation is a symbol system' (Cubitt 1998, p. 31)

On digital shadows: we could just consider digital contexts that simulate the objects, screens and kinetic characteristics of world shadow puppetry traditions, like the work of Lam et al. briefly described below. I assert we should look further afield to the puppetry experimenters, the animators, sculptors and Lumia artists who form and creatively manipulate actual shadows, and their representations via their re-projected image. We should look at a range of symbol systems.

The work of Lam et al. (2008) draws together techniques for real-time simulation of shadow puppets drawn from Chinese traditions. Their paper explores using standard OpenGL techniques of texturing, animation, image processing (depth of field blurring), modelling light and procedural algorithms for (automatic) animation.

From the perspective of performance and puppetry, although real-time image generation techniques were examined, Lam et al. describe no real-time performance system. The paper mentions the potential for real-time physics simulation in creating expressive animation, but doesn't develop or propose any techniques for performer-object control, or enlivening the technical demo through performance by puppeteers. The necessity and execution of traditional puppetry skills are removed from the picture.

The Lam paper (2008) mentions an important and emerging theme of my work, that of preservation of traditional crafts and the importance of digital media preserving and promoting cultural heritage. A theme of the Practice Review and my conclusion is to emphasise the act of performance: What role has performance in transforming a visualisation or a simulation into a pure puppet?

\section{PRACTICE REVIEW}

The practice review selects examples of interactive art with direct relevance to digital shadow puppetry in recognition of contemporaries working in related domains and to acknowledge prior art. The field is expanding and exciting projects proliferate. The commentary to each project will highlight themes as the basis for further discussion. A longer illustrated version of this review is hyperlinked at the end of the current paper.

To steal a phrase from Geiser (2013): our interest in the practice review is "work that is engaged in cutting edge art practices, and [are] in deep conversation with our contemporary culture of simulation and mimicry (digital, robotic and otherwise), while embracing the aura of the handmade and hand-operated." (Geiser 2013)

\subsection{Myron Krueger and Video Place}

Myron Krueger and his collaborators with 'Video Place' starting around 1972, designed nearly fifty interactions (or compositions), each playing with the interaction of silhouettes of differing scales, with objects and interventions from other (often distant) spaces. At once telematic and performative, the system became a technological and aesthetic base-line for future computer vision art installation systems. 
Krueger (1991) describes the system:
"Each participant's video image is digitised and is fed to a series of specialist processors that analyses the resulting silhouettes. These processors analyse each image in isolation (e.g. posture, rate of movement) and with respect to graphic objects and live images on the screen. ... When the participant's actions are understood by the specialised processors, they are reported to the executive processor that decides what the responses should be. Depending on the participant's behaviour, it can move an object, change that objects colour, move the participant's image, or make a sound " (p.44-45, Krueger,1991)

According to Hansen (2006) Krueger's output marks a technical and aesthetic accomplishment, especially in the experience of 'action-response synchronicity':
"Videoplace, Krueger's interactive platform, marks a further and, in some sense, ultimate, stage in the restoration of autonomy to the responsive environment, understood as encompassing ... the embodied visitor. Videoplace works by capturing an image of the visitor's movement, only in this case the image presents the outline of the visitor's body processed (and distorted in various ways) by the computer." (p.35, Hansen, 2006)

We see the responsive environments and embodied visitor as Hansen describes through many of the practice examples.

In both traditional and contemporary shadow puppetry, the property of 'scale' is a subject of play. The scale of the screen, the dynamic scale of the figures, the playful substitutions of scale - the scale of a shadow as we have seen is temporally and spatially dynamic.

\begin{abstract}
"It is as if evolution has prepared us for seeing ourselves on television screens combined with computer images but all, one of the main attractions is the juxtaposition of large and small. So that two people are now interacting and, to some extent discover what the possibilities are and what is suggested emotionally by scale." (Krueger, 2006)
\end{abstract}

As a pioneer in the artistic and technological fusion of camera sensed interactive art, he established the thinking space and vocabulary for embodied, creative interactions yet to come. For example: 'videotouch' refers to the manipulation of a graphic object by touching it and the special sensation felt when 'you touch another person with your image'. He pre-conceived problems still current and germane to digital puppetry, for example: what constitutes true 3D input and how do we overcome the dissociations experienced when hand detection is used as input either 'gloved' or not as with the new 'Leap Motion' hand input device.

Krueger also helped establish the dynamics of realtime interaction and, according to Dinkla, promoted the invisibility or the 'receding' of the interface:

"On account of the synchronicity [of the movement of the video image] with the movement of the [visitor's] body it is no longer a question of distinguishing between the activity of the system and the activity of the visitor. The computer system's role as interaction partner fades into the background, and it now makes itself available as an instrument for the visitor to use." (Dinkla cited in Hansen, 2006, p.36)

\subsection{Jeff Han and The Meme of Multitouch}

Jeff Han, founder of the company 'Perceptive Pixel', led the birth moment of the meme of multitouch surfaces at a TED talk in 2006 ("Jeff Han demos his breakthrough touchscreen"). His demonstrations included two that captured the potential of the technology as an interface for puppeteers.

His commentary, while he draws and animates an object in real-time, emphasises the multiple points of manipulation, the connections to 2D puppetry and the very impressive real-time drawing and mesh deformation routines.

"I can draw out a curve. And when I close it, it becomes a character. But the neat thing about it is I can add control points. And then what I can do is manipulate them with both of my fingers at the same time. And you notice what it does. It's kind of a puppeteering thing, where I can use as many fingers as I have to draw and make." (Han 2006)

Another example from Han, the heat map less obviously related to puppetry, holds a key to more interesting ways of 'controlling' procedural visuals. The dwell-time, the duration of touch, adds or subtracts 'heat' from a 3D fluid simulation, creating a modelling environment and, exciting to the animator, a way of energising 'excitations to movement'. Here l'd like to emphasise the move from direct control of screen objects, to another model of 'indirect' control. This also has led me towards a visual exploration of 'soft-body' simulations as digital shadow puppets (Grant 2013).

The Jeff Han demo illustrates the common decry that the tech-demo hypes the moment, but frequently doesn't yield critical tools for the performer. With tabletop interaction and come the iPad, we do see further exploration of multi-touch puppetry in Grant (2010), Moon (2013), Leite (2011, 2012a, 2012b, 2013) and Leite and Orvalho (2013).

In recent work, Han demonstrates the networked multi-touch environment. As puppetry performance is frequently a collaborative act: with multiple performers controlling a single figure (in tabletop puppetry (short-rod), or Bunraku) or the necessity for multiple objects or figures to be in play, networked multi-touch devices are attractive when creating a digital puppetry performance. The latest work of Grant (2013) and Leite (2013) with Unity3D and 'Brass Monkey' and iPad Open Sound Controllers (TouchOSC), facilitates multi-user, multi-touch digital puppetry performance environments.

\subsection{A Worthers Original: Shadow Monsters}

Philip Worthington's 'Shadow Monsters' (2004, MOMA 2012), created at the Royal College of Art 
on the Interaction Design masters programme has seen life as a story-telling project, an installation and most recently exhibited at the Museum of the Moving Image, New York.

'Shadow Monsters' was created in Processing using the 'Myron' computer vision library (a SourceForge project with the aim 'to keep computer vision free and easy for the new media education and arts community'). Sharp silhouettes created against a light-box were videoed. The live video signal was processed: detecting motion, top and lower edges of the arm and top and lower edges of the hand-shape. Random teeth, hair, eyes, fur, fins and spikes were generated and the resultant augmented shadow image re-projected to a screen for the performer and audience to see. Sounds were generated in sympathy with the pace of arm movements and with regard to the 'mouth' motion.

Through many different configurations and iterations of the project, the MOMA exhibit led to a separation (in distance) of the white light-box screen, used to enhance the silhouette and improve the computer vision performance and the screen displaying the augmented image.

Stylistically, the augmented images of 'Shadow Monsters' resemble the actual object play in the experimental shadow theatre of Schönewolf (1969). However, the generative graphics and sound forego the tactility and assemblages of object play and lead to a disrupted 'proprioception' for the interactant/performer. The proprioception of the body (of the performer) and the body-in-image when re-projected is an additional peripheral awareness and represents a disconnect. Such a spatial/physical disconnect is familiar to puppeteers. Consider muppeteer Frank Oz operating 'Miss Piggy' above his head while watching an external camera view of his own actions on a reverse scan monitor below his feet).

\subsection{Golan Levin: Computer Vision for Artists}

Golan Levin, the prolific interactive artist and educator, is producing a range of work that resonates with the field of puppetry: including augmented shadowgraphs, sonified shadows, automata and robotic sculptures. His work teaching and popularising computer vision techniques for artists follows and builds on the legacy of Myron Krueger and others. Computer vision technologies:

(6) bring the silhouette to the centre (symbolically) of a range of modalities of interaction, and

(7) facilitate (dis)embodied interactive experiences and the whole range of puppeteering like behaviours users, interactants, digital puppeteers exhibit with electronic forms (N.B. not exclusively digital forms).

In the 'Interstitial Fragment Processor' we see one of a range of iterations of an interest in the sonic and visual properties of phenomena Levin refers to as phonesthesia, or sound symbolism. In this example, the negative space created by shadows and silhouettes form elastic shapes that drop under the control of simulated physics and generate sounds on impact with the ground plane and with other spaces.

The augmented human shadowgraph is central to the visual space of the installation. Collaborations within and between shadows (and people) are elicited. The game generates shapes that proliferate on the screen.

\section{Levin and Lieberman write:}

"We conclude that our instruments, which merge real-time sound with virtual synthetic graphics and organic analog shadows, enable a new form of live audiovisual cinema to be performed in the hybrid locale of an augmented reality." (Levin \& Lieberman 2005)

In both projects we see a division between playspace, screen and spectator. The player spectates on their own shadow, whilst in the grip of the game and movement (and in the case of 'Messa Di Voce', sonic) improvisations.

To get a sense of the movement and visual augmentation in 'Messa Di Voce', here are descriptions from the documentation:
Vignette 6: Insect Nature Show: "In this module, Joan's silhouette is augmented by an interactive projected shape. This black shape is a larger, bouncier version of her own outline. The edge of this shape, moreover, also changes in response to her speech: it develops wiggles which reflect the timbre and pitch of her voice." (Levin et al. 2004)
Vignette 4: Bounce (Jaap's Solo). "A man enters an empty white void. He emits a stream of bubbles by making a special cheek-flapping sound. As his sounds grow more vigorous, his bubbles fill up the screen. But the resulting cloud of jostling sound-bubbles is unstable. Turning to admire his work, his cloud bursts-raining bubbles that, when they fall onto him or crash to the ground below, replay recordings of his cheeky sounds. He tries to contain the noisy torrent, but, failing this, storms off in distress." (Levin et al. 2004)

There are several moments, particularly where the players play towards the screens, not the audience, where the technical constraints of camera tracking the performers against a high contrast background over-determining the staging: a bit like watching someone use an iPad, albeit, on a bigger scale. The act of image occlusion by an interactant and subsequent re-direction of audience attention is a problem. The visual illusion, though, of mapping generative augmented shadows, objects, particle systems, placards, to moving performers is effective. Having the penumbra of shadows modulated by sound and voice brings a sensate awareness of the performers to their own shadows. An idea significantly explored in Worthington's 'Shadow Monsters'.

"Owing to the head-tracking system, moreover, these visualisations can be projected such that they appear to emerge directly from the 
performers' mouths. In some of the visualisations, the projected graphical elements not only represent vocal sounds visually, but also serve (bidirectionally) as a playable interactive interface by which the sounds they depict can be re-triggered by the performers." (Levin et al. 2004)

\subsection{Miwa Matreyek 'Dreaming of Lucid Living'}

Miwa Matreyek, an experimental animator and multimedia artist, came to widespread recognition at a 2010 TED talk in Oxford. Her piece "Dreaming of Lucid Living" (2009) is a carefully choreographed interaction between projection mapped animated media and the performers shadowgraph. At various times we see in silhouette the full body, head and hands, with a variety of physical props and virtual adornments. On the style and flow of the piece: the separate vignettes and segments are stylistic of much shadow theatre. The colour-scape mimics the greyscale palette of shadow theatre, with lavish splashes and crescendos of colour as the piece progresses.

At times, the performer plays with space both in front of and behind the screen. The silhouette as a semi-illuminated relief integrates with animated actions becoming part of the montage and, when behind the screen, the darkness is augmented with projections mapped within the boundaries of the silhouette. The piece is a digital extension of a shadowgraph or ombromanie performance with it's careful synchronisation of recorded sound, image and movement. It can be viewed in direct contrast to the chaotic real-time responsive installations of 'Messa Du Voce' or 'Puppet Parade.'

The work is a choreographic scenography, where the human role plays bit-part to the flow and mapping of animated images. Though not totally effaced, the performer eventually breaks free of the surface, transgresses the image boundaries and builds a projection-mapped cityscape.

\section{Like nineteenth century shadowgraphy:}

\begin{abstract}
"the virtuoso shadowgraph performance did not efface the performer positioned behind the screen in order to intensity the projected fictions but rather emphasised the performative nature of the screen images in order to continually remind the spectator of the shadowgraphist's very presence." (Solomon 2000, p.13)
\end{abstract}

\section{6 lan Grant and the 'ShadowEngine'}

The author's current doctoral project is evaluating in performance and with interactants (puppeteers, audiences, animators and programmers) the expressive qualities of different modes of interactive digital puppetry, from multitouch surfaces, tablets to tabletops, to physical sensors, to body control camera vision techniques using the Kinect and whole hand detection with the Leap Motion. For this purpose, l've created new performance animation systems and software. With one such prototype application the 'ShadowEngine' running on a multitouch portable device, the Apple iPad.
To this end, we perform real-time animations paying homage to traditional world shadow performance forms and animated film. Currently we have scenes drawn from Karagiozis, figures from Indonesian Wayang Kulit, from animation, a character in homage to silhouette film maker Lotte Reiniger and an extended piece in celebration of the Fleischer studios.

The 'ShadowEngine' makes multi-touchable digital shadow theatre possible using physics based realtime animation. The characters and objects are curiously 'expressive', and the performance analysis begins to refine the issues involved when operating and viewing complex multi-jointed characters in a physics-based 3D (pseudo 2D) environment.

The primary aim was to prove the basic concept: that the 3D models, with UV and texture maps, could work in an orthogonal openGL context, simulating the physical interactions of shadow puppets in a multitouch environment. There is a rationale behind each of the figures and they all make it to the screen through different design processes. Each character has a different rig and configuration of joints. All the objects are built of 'rigid-body' parts and have different physical properties - with the configurable joints, hinges, mass, spring and damping properties all varied and nuanced.

It is satisfying that the proof of concept works. Playing can lead to 'operator emoting', performer flow and rich expressive moments. In terms of performance animation, the combination between direct control and physical simulation (and it has to be said 'accidental physics glitches') is a ripe area for further exploration. The focus on traditional forms is a starting point for me. The act of simulating old forms without obvious visual transformation in a digital context is neither 'farcical, futile' but 'deeply fertile.' to paraphrase Druckrey quoted earlier.

\subsection{Luís Leite's Virtual Marionettes}

Luís Leite's 'Virtual Marionette' projects (20112013), currently part of a doctoral study, have their main focus in pedagogy, though the various demos have clear performance and installation applications. Seeking a simpler way for children to interact with puppetry, Leite has explored a variety of multimodal interfaces including the Kinect, multitouch surfaces (the iPad) and the Wii remote. In his multitouch project Leite makes great use of the software 'Animata' and the iPad - to send Open Sound Control (OSC) messages to send multiple touch points to transform the joints of a shadow puppet, built in 'Animata'.

'Animata' facilitates the setup of 'as-rigid-aspossible shape manipulation' technique (seen in the Jeff Han 2006 demo) and can also simulate jointed rigid-bodies, but not the physical environment.

Luís Leite's 'O Pássaro da Alma' is one of the more interesting efforts to map the humanoid skeletal data from OpenNITE (the open Kinect project) to 
non-human figures. The interest in 'indirect' mappings creates a very different style of interface 'language' and also presents animation issues. The novel interface language means different control body shapes need to be explored, through play, to discover the characters effective poses and qualities of movement.

Animation issues include over-lapping elements and order-sorting issues, gestural glitches or physics 'freak-out'.

Using a silhouette is a practical convenience as the blackness, 'the material density', of the shape excludes confusion about the physical reality of the movement and problems like self-occlusion disappear.

The project is a great example of what becomes interesting in whole body interactions where the mechanics of the body are 'remapped' in more-orless arbitrary ways to the object of animation.

\section{EMERGING THEMES AND ISSUES}

\subsection{Performance}

We need to advocate pulling impressive demos out of the lab and into performance. This may be by pursuing the technologists with requests to use their tools and then collaborating or making tools that emulate the same kind of outcome.

\subsection{Animation Qualities: Simulation, Chaos and Accident}

In the practice review (larger in the full paper) there is a divide between digital puppetry graphic environments that use physics simulation to act upon the performance objects and those that do not (and one - the Fluid Interface Group at MIT who blend both). It will be a point of future evaluation of the nuances of expression that both facilitate.

A theme emerging from a number of projects is the combination of animation that is:

(i) Driven by user interaction;

(ii) Driven by objects under physics simulation (e.g. gravity on joint chains or flocking algorithms);

(iii) Driven by pre-made and blended with simulated animation;

(iv) Automated using generative non-planned techniques (behavioural simulation);

In a future study analysis of the qualities of animation will relate the 'expressivity' of a moving object with its 'liveliness'. By liveliness I refer to the analytical framework of motion perception in the work of Rudolf Arnheim. Chow (2012) usefully places Arnheim into the context of animation and Eastern traditions (a connection to the major shadow puppetry cultures):

"For Arnheim, what counts as liveliness is not whether there is really a mind or soul, but rather the level of complexity in the observed behaviour. He delineates different degrees of liveliness according to different levels of complexity, from simple movement to complex behaviour, as shown in the following list: 1.
Something that moves is livelier than something that does not. 2. Movement involving internal change (i.e. change in shape) is at a higher level of complexity than rigid object displacement. 3 . The thing moving by its own force (i.e. selfinitiated movement) is higher in degree of liveliness than that physically moved by others. 4. Those self-movements initiated by internal impulses are livelier than those driven by external forces." (p.178, Chow 2012)

It is movement emerging from the 'automatic' that carries expressive potential (or liveliness). Control should begat the opportunity for the accidental.

\begin{abstract}
"Naturally, as a manipulated object, the puppet can only begin to approach real being by detaching itself from any external control. Its first task is therefore to capture the sense of naturalness implied by freedom of movement. For Kleist movement is natural when it is automatic; habitual but not willed. The natural is a mechanical response, and the puppet is perfectible because it has a memory but no will. In this line of thinking the mechanical and the natural are matching towards some sort of cybernetic union where mechanical perfection means not only the absolute reproduction of the real, but freedom from accident." (Paska 2012, p.139)
\end{abstract}

It is through certain control methodologies of digital puppetry a 'cybernetic union' can be formed. My hypothesis is: expressive potential is unlocked when a combination of direct control, simulation and automated techniques are blended.

\section{CONCLUSIONS}

(1) The puppet (as an artefact and metaphor) is a rich conceptual tool for understanding the expressive potential of cybernetic, interactive media forms; Some points from Roman Paska:
"Having no interior life...the puppet is, strictly speaking, incapable of expression. Having nothing to express, it can express nothing. The mask of an actor or dancer, conceals the density of humanity; the puppet, nothing but emptiness. Nothing. What is the Nothing that hides behind the mask of the puppet? What is the puppet stripped bare? The fascination of the puppet, its secret power of seduction, lies in what it hides, not what it expresses. Narrative, mimesis, representation: all 'orthodox' aspects of puppet theatre in the West. Little human simulacra illustrating human quirks through the imitation of human poses and gestures. Mimicry and parody as the twin peaks of the puppeteer's art." (Paska 2012, p.136-137)

The nothing of the physical puppet maps nicely onto the zero of the digital puppet.

(2) Using innovative human interface devices, such as the Kinect, the Leap Motion and multi-touch surfaces, and harnessing their expressive potential, are acts of pure puppetry;

On a conceptual level, computers are objects, the code that in all likelihood sequences our digital experiences are also known as or create objects: getting real and natural in our embodied interactions with and manipulation of machines is puppetry. 
(3) Puppetry is a rich performance practice (in all it's various physical forms) ripe for computer simulation;

I would add: the hybrid form of puppetry is also ripe to be transformed by new computational practices. As long as it remains in the mode of performance, is tested by means of performance, and if begat through performance. Make the move from the lab, from the demo, to the performance space, where as Paska has it, all reckoning begins.

"The puppet-as-object in live performance still represents the 'zero degree' of puppetry (the point from which all reckoning begins)." (Paska 2012, p.139)

In conclusion, the collection of practice considered in this paper unites to tell a story of the continuation of shadow theatre aesthetics in the post digital age. Whether the motivation is a retro interest in the analogue or a more fundamental attraction to the interplay of darkness and the luminescent in a quasi-Platonic or immaterial domain, is the subject of further investigation.

"The power of such images-the silhouette, and the black-and-white portrait photograph-arises from their origin in the light that once played on their subjects and formed their image. They are emanations, captured and stilled. Is that a figure of speech? They are copies of the originals, and in that sense, their character ceases to be metaphorical. It is here, on this edge where the figurative touches the actual and the image becomes reality, that shadow eerily communicates individual presence; this effect grows when a shadow becomes a shade, and that shade a reflection; then the projected image of a person brushes the condition of spirit." (Warner 2007)

A version of this paper with full illustrations and an extended practice review can be found at: http://www.daisyrust.com/eva2013/

\section{REFERENCES}

Chow, K. K. (2012) Toward Holistic Animacy: Digital Animated Phenomena echoing East Asian Thoughts. Animation, 7(2):175-187.

Cubitt, S. (1998) Digital Aesthetics. Theory, Culture \& Society Series. SAGE, London.

Digital Arts Week. (2007) Digital Art Weeks Call for Participation.

http://www. digitalartweeks.ethz.ch/web/DAW07/Front (retrieved 17 April 2013).

Druckrey, T. (2008) Foreword. In Zielinski, S., Deep Time of the Media: Toward an Archaeology of Hearing and Seeing by Technical Means. MIT Press.

Geiser, J. (2013) Janie Geiser - Automata. http://www.janiegeiser.com/section/automata. (retrieved 17 April 2013).

Grant, I. (2008) Experiments in digital puppetry: Video hybrids in apple's quartz composer. In Adams, Randy. Gibson, S. and Muller-Arisona, S., editors, Transdisciplinary Digital Art: Sound, Vision and the New
Screen. Springer-Verlag Berlin and Heidelberg $\mathrm{GmbH}$ \& Co. K.

Grant, I. (2013) Digital performance practice and research. http://www. daisyrust.com (retrieved 17 April 2013).

Han, J. (2006) Jeff Han demos his breakthrough touchscreen | Video on TED.com. http://www.ted.com/talks/jeff_han_demos_his_breakthro ugh_touchscreen.html (retrieved 17 April $201 \overline{3}$ ).

Hansen, M. B. N. (2006) Bodies in Code: Interfaces with Digital Media. Routledge, New York.

Kaplin, S. (1999) A puppet tree: A model for the field of puppet theatre. TDR (1988-), 43(3):28-35.

Krueger, M. W. (1991) Artificial Reality II. AddisonWesley, Reading, Mass.

Krueger, M. W. (2006) Myron Krueger - Videoplace, Responsive Environment, 1972-1990s. [online video]. http://www. youtube.com/watch?v=dmmxVA5xhuo (retrieved 17 April 2013).

Lam, T. K., Talib, A. Z. b. H., and Osman, M. A. (2008) Real-Time Visual Simulation and Interactive Animation of Shadow Play Puppets Using OpenGL. World Academy of Science, Engineering and Technology, (45):212-218.

Leite, L. (2012a) IPADATA - digital puppetry on vimeo. https://vimeo.com/30826473 (retrieved 17 April 2013).

Leite, L. (2012b). Virtual marionette. In Proceedings of the $2012 \mathrm{ACM}$ international conference on Intelligent User Interfaces, IUl'12, pages 363-366, New York, NY, USA. ACM.

Leite, L. (2013). Virtual marionette. http://www.grifu.com/vm/ (retrieved 17 April 2013).

Leite, L. and Orvalho, V. (2011). Anim-actor: understanding interaction with digital puppetry using lowcost motion capture. In Proceedings of the 8th International Conference on Advances in Computer Entertainment Technology, ACE '11, pages 65:1-65:2, New York, NY, USA. ACM.

Leite, L. and Orvalho, V. (2013). Inter-acting: Understanding interaction with performance-driven puppets using low-cost optical motion capture device. International Journal of Advanced Computer Science, $3(2)$

Levin, G. and Lieberman, Z. (2005). Sounds from Shapes: Audiovisual Performance with Hand Silhouette Contours in The Manual Input Sessions. In Proceedings of NIME '05, Vancouver, BC, Canada., Vancouver.

Levin, G., Lieberman, Z., Blonk, J., and La Barbara, J. (2004). Messa Di Voce Project Report. [online] http://www.flong.com/storage/pdf/reports/messa_report_ 600.pdf (retrieved 17 April 2013).

Moon, J. and S.H. Nam (2010). Augmented Shadow Joon Moon - Portfolio. http://joonmoon.net/AugmentedShadow (retrieved 17 April 2013).

Paska, R. "Notes on Puppet Primitives and the Future of an Illusion" in Francis, P. Puppetry: a reader in theatre practice. Palgrave Macmillan, 2012.

Warner, M. (2007). Darkness Visible. In Cabinet Issue 24: Shadows [online] Available from: http://www.cabinetmagazine.org/issues/24/Warner.php (retrieved 17 April 2013). 